\title{
An Innovation of an Academic Cloud Computing Service
}

\author{
Wen-Lung Shiau ${ }^{1}$, Han-Chieh Chao ${ }^{2}$, Chia-Pin Chou ${ }^{1}$ \\ ${ }^{1}$ Department of Information Management, Ming Chuan University, Taoyuan County, Taiwan; ${ }^{2}$ Institute of Computer Science \& \\ Information Engineering and Department of Electronic Engineering of National Ilan University, I-Lan, Taiwan. \\ Email:mac@mail.mcu.edu.tw, hcchao@gmail.com, chiabin.chou@gmail.com
}

Received August $16^{\text {th }}$, 2012; revised September 18 ${ }^{\text {th }}$, 2012; accepted September $27^{\text {th }}, 2012$

\begin{abstract}
Despite the popularity of cloud computing services, few studies have investigated academic cloud computing services. This study explored the innovation of an academic cloud, the Ming Chuan University Cloud System (MCUCS). A cloud computing service provides numerous benefits to an organization. We conducted a comprehensive review and observation of MCUCS and identified 8 benefits for teachers and 10 for students. Teachers and students agreed that MCUCS consistently provided a service that improved their performance. The most beneficial features of MCUCS were its high usage and user satisfaction. Between April 2010 and April 2012, the usage exceeded 878,300 events. Overall student satisfaction with MCUCS was $79.2 \%$, and teacher satisfaction was $86 \%$. We concluded that MCUCS provides a good model of an academic cloud computing service and a high qualified system.
\end{abstract}

Keywords: Innovation; Academic Cloud Computing Service; Evaluation; Benefits; Satisfaction

\section{Introduction}

During the past decade, information technology (IT) has become increasingly popular and the Internet has flourished. People regularly obtain required information and knowledge from the Internet, both for work purposes and in general life. This dependence on the network has resulted in a focus on cloud computing. However, several market analysts and companies have expressed concerns about cloud computing, which as a field is expected to reach maturity within the next 4 to 6 years $[1,2]$. According to CNN [3], a survey of approximately 900 Internet and technical experts and social analysts showed that the majority of Internet users will "live mostly in the cloud" by 2020. Among the experts surveyed, $71 \%$ predicted that people will operate primarily through webbased and mobile applications (apps), with far less reliance on software installed on desktops.

Cloud computing is a service rather than a product, whereby shared storage resources, software, and information are provided to computers and other devices on demand. The service operates in a similar manner to other utilities, such as the electricity grid, over a network (typically the Internet), without cloud users needing to know the location of the computing infrastructure or other details thereof $[4,5]$. According to the United States National Institute of Standards and Technology (NIST), the main characteristics of cloud services are: on-demand self-service, broad network access, location-independent resource pooling, rapid elasticity, and measured service. Cloud computing providers offer their services through 3 fundamental models: Infrastructure as a service (IaaS), platform as a service (PaaS), and software as a service (SaaS) $[4,6]$. Figure 1 shows the components of cloud computing service.

Because computation occurs on a remote server, the users' hardware requirements remain minimal. Thus, cloud computing reduces both cost and maintenance requirements [7]. This benefit has led school administrators seeking to reduce IT budgets to focus on cloud computing services [8]. Gartner [9] reported that more than 2000 CIOs identified cloud computing as a top technology priority for 2011. Other studies have predicted that the global cloud computing market will increase from $\$ 40.7$ billion in 2011 to \$241 billion in 2020, and that SaaS will be adopted by companies of all sizes in the future. These studies predicted that in 2011, SaaS would constitute a $\$ 21.2$ billion market, which would grow to $\$ 92.8$ billion by $2016[1,10]$. Such predictions suggest that numerous companies are starting to use cloud computing to improve their effectiveness.

\begin{tabular}{|c|}
\hline Software as a service (SaaS) \\
\hline Platform as a service (PaaS) \\
\hline Infrastructure as a service (IaaS) \\
\hline
\end{tabular}

Figure 1. Cloud computing service components. 
Since the mid 1980s, educational experts have been speaking of "virtual classrooms". In a virtual classroom, people who are spatially and/or temporally distant from each other can be involved in collaborative learning processes using technological systems that reduce time and space constraints [11,12]. Virtual classrooms provide additional advantages such as superior mastery of course material compared with that provided by traditional classroom settings. Virtual classroom students report higher subjective satisfaction with virtual classrooms than traditional classrooms on a number of dimensions, including access to professors and the overall quality of the educational experience. Furthermore, virtual students perceive the experience as "group learning" rather than individual learning. The more students perceive collaborative learning, the more likely they are to judge the outcomes of a virtual classroom as superior to those of a traditional classroom [13,14].

An academic cloud computing service supports both virtual and traditional classrooms, and assists both teachers and students in improving their performance. However, few studies have investigated the phenomenon of educational cloud computing. This study explored the innovation of an academic cloud computing service. The remainder of the paper is organized as follows: Section 2 describes the Ming Chuan University Cloud System (MCUCS), and Section 3 discusses the evaluation of MCUCS. The final section presents our conclusions.

\section{Ming Chuan University Cloud}

The term "cloud computing" refers to software applications or resources that exist online and are available to multiple users through the Internet, without having to be installed on a user's computer. Users can access these applications from any computer with an Internet connection, without needing any other connection to the hardware that holds the source software [15]. This system is attractive in its potential for developing computing technology and because of its benefits for learners and teachers. The cloud system provides educational flexibility and reduces the cost of providing IT education.

The MCUCS was created by 8 cores of processing power, 48 GB Ram, 28 blade servers, 25 TB storage, 240 virtual machines (VM), and Windows Server 2008 R2. It comprises 3 subsystems: cloud classroom, cloud folder, and cloud host (Figure 2) and offers SaaS and IaaS.

Up to 900 students can simultaneously use the MCUCS. Students, teachers, and other employees need to authenticate their identification before using multiple resources.

\subsection{Software as a Service (SaaS): Cloud Classroom}

SaaS provides the use of applications linked to a cloud

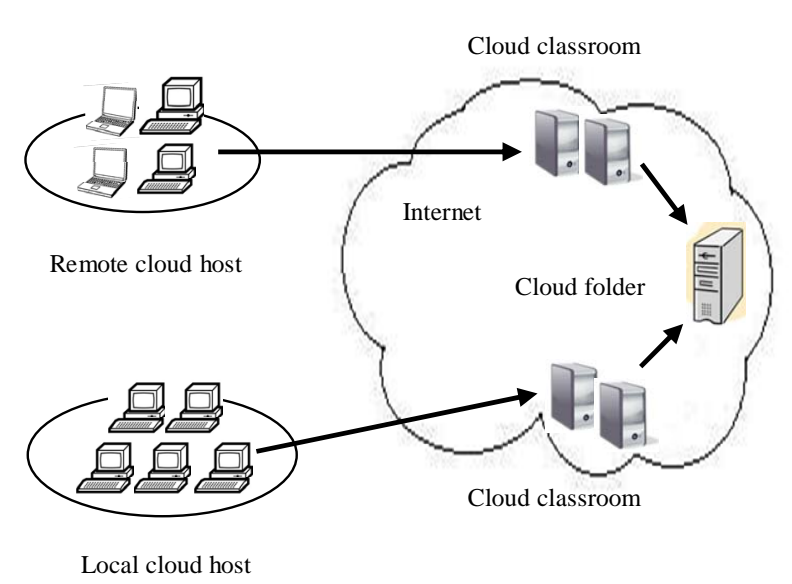

Figure 2. Ming Chuan University cloud infrastructure.

system. The applications are accessed by client devices, either through a thin client interface such as a browser (e.g., web-based email) or a program interface. The end users do not manage or control the underlying cloud infrastructure (network, servers, operating systems, storage, or individual applications). However, certain applications allow for limited user-specific configuration settings [4,5]. The cloud classroom provides educational software, Technicians Quotient Certification training files, Microsoft Office, Adobe Creative Suite, programming tools, and certain specialized applications such as statistical software (SPSS or SAS) and ERP (SAP or Workflow ERP). Figure 3 shows the screen of cloud classroom. Teachers are able to record a classroom session and save it in a teaching file, which students can then access at any time and place, as in a virtual classroom.

The cloud classroom also provides virtual space for users to store their files. Each user receives $4 \mathrm{MB}$ for a system disk (C drive) and 2 GB for a file disk (D drive), as shown in Figure 4. Users can thus access the resources off-campus from a fixed computer environment.

\subsection{Infrastructure as a Service (IaaS): Cloud Folder}

IaaS provides computer infrastructure. This mode offers products that are delivered by remote service through the Internet, and includes a full computer infrastructure (e.g., virtual computers, computational resources, and data storage facilities). The best-known vendors of such services include Amazon's EC2, GoGrid's Cloud Servers, and Joyent.

Transferring files can pose a problem for learners and teachers, and the cloud folder was developed as a solution to this problem. When users login to the system, a popup message prompts: "You can load your personal cloud folders (Desktop, My Documents, Favorites, My 


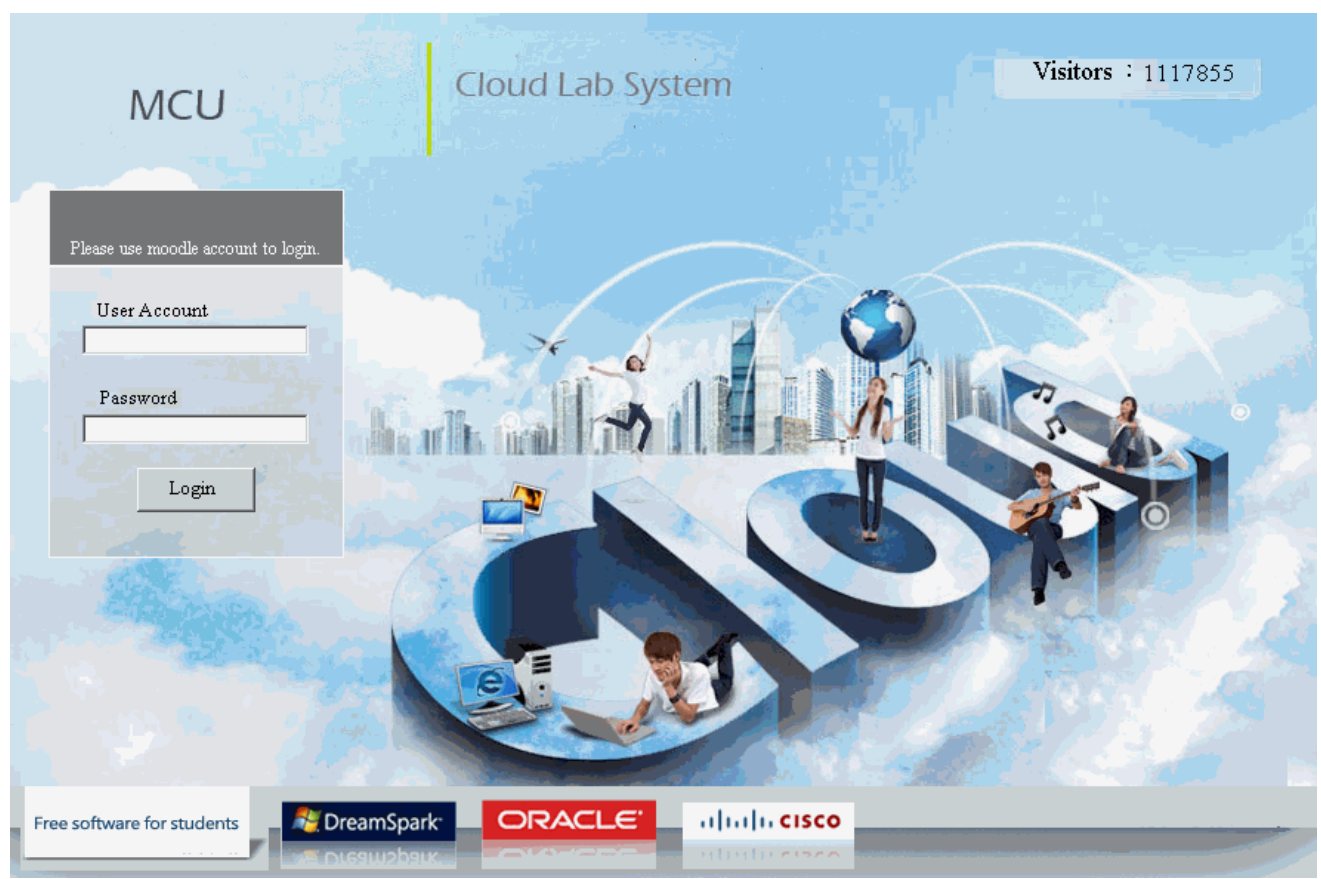

Figure 3. The screen of cloud classroom.

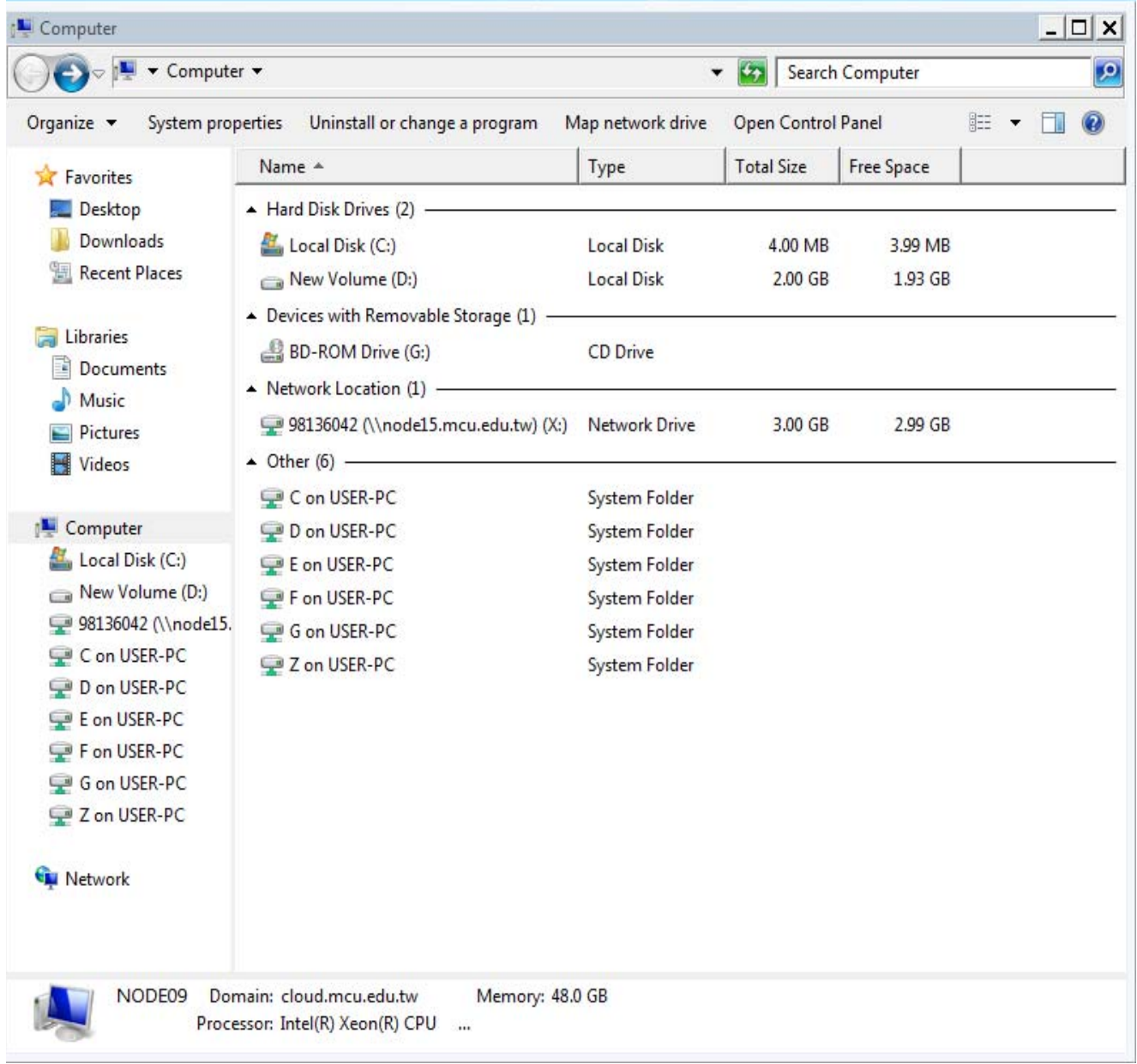

Figure 4. The cloud folder of Ming Chuan University Cloud System. 
Music, My Pictures, My Video, and Downloads) to this computer. Do you want to load cloud folders?” Each user receives 3 GB of free space (network disk, shown in Figure 2) in which to centrally store their frequently used files. All they need do is click the "Yes, please load cloud folders” button.

\subsection{Infrastructure as a Service (IaaS): Cloud Host}

The cloud host allows a user to rent a free virtual computer to run his or her specific computer applications. Each user can rent only one cloud host. Students can rent it for a month; teachers and other personnel can rent it for 6 months. Borrowers can renew their projects before the rental time expires. Users are required to adhere to the rules of the cloud host and project management.

The cloud host provides a scalable deployment of applications by providing a Web service, through which a user can boot a machine image to create a virtual computer. This "machine" comprises an operating system (Windows 7, Linux, and FreeBSD) and any software desired. Figure 5 shows an example of a cloud host project. Figure 6 shows the operation of booting a cloud host.

\section{Evaluation of Ming Chuan University Cloud System}

The MCUCS is a large and complex system. Developing such an extensive academic cloud system can cost a great deal-up to millions of dollars. The MCUCS is constrained by fixed costs for hardware and software development. Thus, the system managers are concerned with understanding the system's perceived benefits. The benefits of the MCUCS for teachers have been identified by school managers as follows.

- Flexibility: Teachers use an electronic document system at school, at home, or at any where.

- Reduced cost: Teachers use free software.

- Reduced workload: The software environment is consistent for teaching and preparation.
- Easy to use: Using the cloud system makes teachers' jobs easier.

- Convenient: The cloud system removes the need for flash drives.

- Improved performance: The cloud system enhances teachers' effectiveness.

- Highly automated: Teachers do not need to worry about making constant software upgrades (versions) to the operating system.

- Increased storage: Teachers can store more data than on a personal computer.

The benefits of MCUCS for students have been identified as follows.

- Reduced cost: Students use free software at school and home.

- Enhanced learning: Students have vast opportunities to try various applications.

- Focus on lessons: Students who prepare software certifications do not need to install software or worry about the software environment.

- Easy to use: Uploading and downloading of files is relatively easy to transfer files.

- Convenient: Exchanging messages with other users of the cloud system is convenient.

- Time-effective: Students can use the cloud system to do homework timely.

- Compatible: The cloud system is compatible with all aspects of the student's personal computer.

- Controllable: The student has full control over his or her use of the cloud system.

- Individual effect: Using the cloud system tends to improve students' grades.

- Group effect: Groups of students work jointly on projects in the cloud system.

This wide range in perceived benefits means that managers are inevitably concerned to evaluate a cloud system. Our evaluation of the MCUCS showed that its most positive features were high levels of usage and user satisfaction. Between April 2010 and April 2012, users logged in more than 878,300 times. A recent survey conducted by

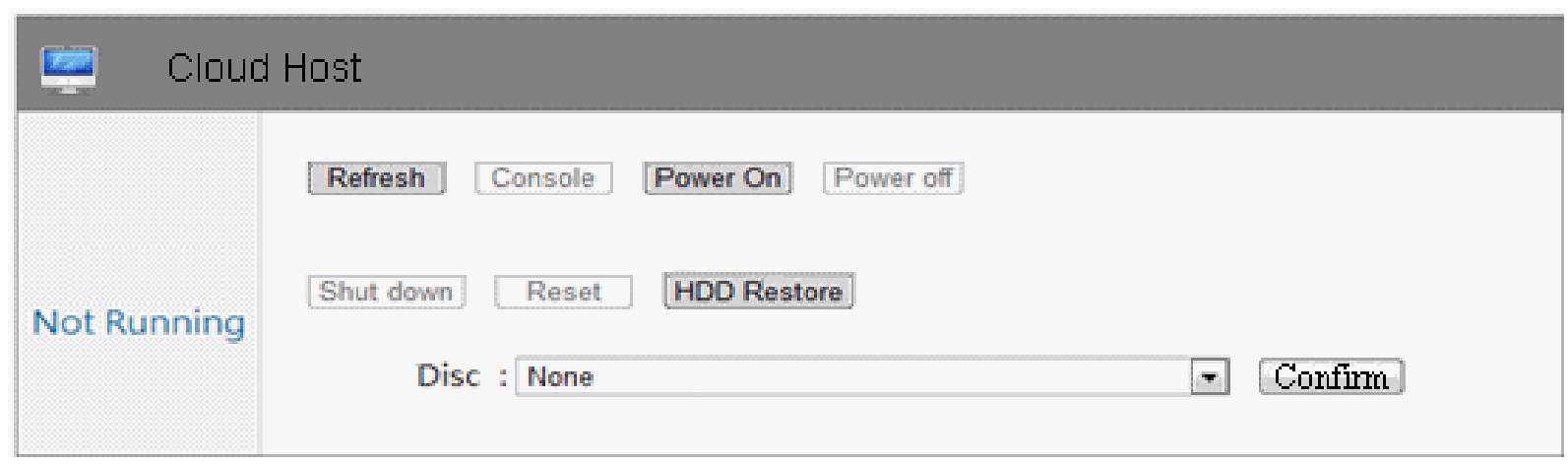

Figure 5. The project of a cloud host. 


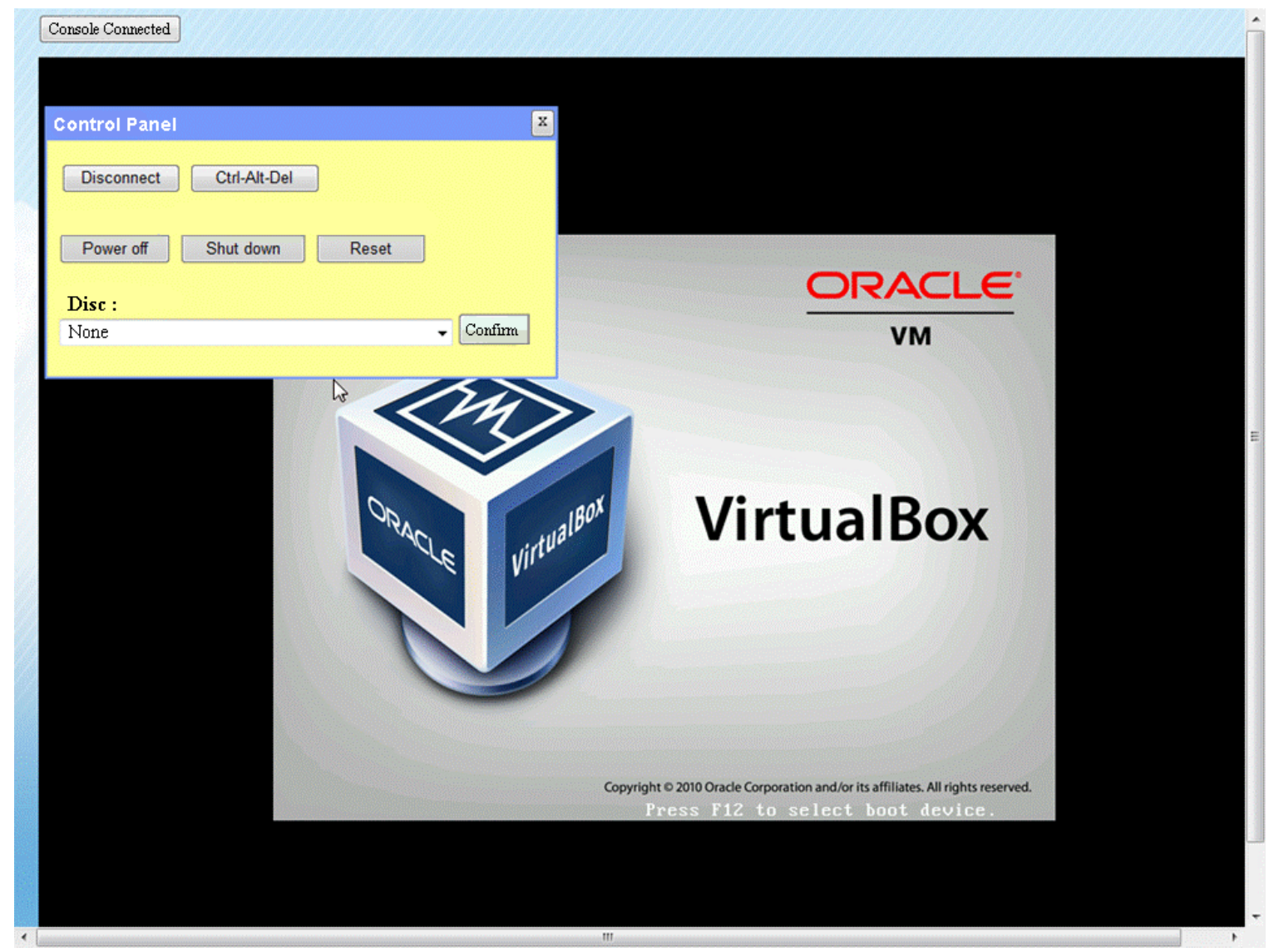

Figure 6. The operation of booting a cloud host.

school managers on MCUCS showed that overall student satisfaction was $79.2 \%$ and teacher satisfaction was $86 \%$. Thus, we concluded that the MCUCS provides a high quality cloud service.

\section{Conclusions}

The MCUCS provides virtual classroom support for dayto-day operations and students' learning activities. This cloud system mainly offers SaaS and IaaS services. SaaS includes educational software, Microsoft Office, Adobe Creative Suite, programming tools (Java), statistical software (SPSS, SAS), and ERP (SAP, Workflow ERP). IaaS includes the provision of a cloud folder and host. The MCUCS provides 8 main perceived benefits to teachers, and 10 main perceived benefits to students. Our overall evaluation of MCUCS was a high-quality system that is popular and well recognized by both teachers and students.

\section{Acknowledgements}

The authors thank Prof. Wang J. L., Professor Abel Chen and Mr. Joseph Huang for providing necessary information of Ming Chuan University Cloud system for this study.

\section{REFERENCES}

[1] S. Ried, H. K. P. Matzke, A. Bartels and M. Lisserman, "Sizing the Cloud: Understanding and Quantifying the Future of Cloud Computing," 2011. http://www.forrester.com/Sizing+The+Cloud/fulltext/-/ERES58161?objectid=RES58161

[2] B. Martens and F. Teuteber, "Decision-Making in Cloud Computing Environments: A Cost and Risk Based Approach,” Information Systems Frontiers, 25 July 2011, pp. 1-23. doi:10.1007/s10796-011-9317-x

[3] J. O'Dell, "Experts Say We'll Be Working in the 'Cloud' by 2020,” 2010.

http://edition.cnn.com/2010/TECH/web/06/11/cloud.mas hable/

[4] P. Mell and T. Grance, "The NIST Definition of Cloud Computing,” National Institute of Standards and Technology Special Publication 800-145, Department of Commerce, Gaithersburg, 2011.

[5] Wikipedia, “Cloud Computing,” 2011. http://en.wikipedia.org/wiki/Cloud_computing

[6] W. Voorsluys, J. Broberg and R. Buyya, "Introduction to Cloud Computing,” In: R. Buyya, J. Broberg and A. Goscinski, Eds., Cloud Computing: Principles and Paradigms, Wiley Press, New York, 2011, pp. 3-41.

[7] C. Erenben, "Cloud Computing: The Economic Imperative,” ESchool News, Vol. 12, No. 3, 2009, pp. 12-19. 
[8] T. S. Behrend, E. N. Wiebe, J. E. London and E. C. Johnson, "Cloud Computing Adoption and Usage in Community Colleges,” Behaviour \& Information Technology, Vol. 30, No. 2, 2011, pp. 231-240.

doi:10.1080/0144929X.2010.489118

[9] Gartner, "Gartner Executive Programs Worldwide Survey of More Than 2,000 CIOs Identifies Cloud Computing as Top Technology Priority for CIOs in 2011,” 2011. http://www.gartner.com/it/page.jsp?id=1526414

[10] L. Dignan, “Cloud Computing Market: \$241 Billion in 2020,” 2011.

http://www.zdnet.com/blog/btl/cloud-computing-market241-billion-in-2020/47702

[11] F. Papa, M. Perugini and S. Spedaletti, "Psychological Factors in Virtual Classroom Situations: A Pilot Study for a Model of Learning through Technological Devices,"
Behaviour \& Information Technology, Vol. 17, No. 4, 1998, pp. 187-194. doi:10.1007/s10796-011-9317-X

[12] S. R. Hiltz, “The "Virtual Classroom': Using ComputerMediated Communication for University Teaching,” Journal of Communication, Vol. 36, No. 2, 1986, pp. 95-104. doi:10.1111/j.1460-2466.1986.tb01427.x

[13] S. R. Hiltz, "The Virtual Classroom: Learning without Limits via Computer Networks,” Ablex Publishing Corporation, Norwood, 1994.

[14] S. R. Hiltz and B. Wellman, "Asynchronous Learning Networks as a Virtual Classroom," Communications of the ACM, Vol. 40, No. 9, 1997, pp. 44-49. doi:10.1145/260750.260764

[15] E. Knorr and G. Gruman, "What Cloud Computing Really Means,” 2011.

http://www.infoworld.com/print/34031 